\title{
Advances and prospects in adoptive cell transfer therapy for ovarian cancer
}

\author{
"T-cell accumulation in epithelial ovarian cancer is prognostic for \\ survival and recent advances in adoptive T-cell therapy now provide \\ a renewed opportunity to specifically modulate T-cell number and \\ activity in epithelial ovarian cancer and mediate tumor regression."
}

Keywords: adoptive T-cell therapy $\bullet$ chimeric antigen receptor $\bullet$ gene engineering $\bullet$ immunotherapy • ovarian cancer $\bullet$ TCR $\bullet$ TILs

Despite recent advances in targeted therapies, epithelial ovarian cancer (EOC) remains the most lethal form of gynecologic malignancy. Thus, improving the clinical outcome of patients will require reinvestment in research development and further clinical investigation. T-cell accumulation in EOC is prognostic for survival and recent advances in adoptive T-cell therapy now provide a renewed opportunity to specifically modulate T-cell number and activity in EOC and mediate tumor regression. Here, we discuss the application of adoptive T-cell transfer based immunotherapy and its prospects for the treatment of EOC.

Evidence of the role of the immune system in human EOC comes from epidemiologic and clinical data demonstrating the presence of $\mathrm{CD}^{+}$tumor-infiltrating $\mathrm{T}$ lymphocytes (TILs) and its association with favorable prognosis and longer survival [1]. Patients whose tumors contained TILs had 5 -year overall survival rate of $38 \%$, whereas patients whose tumors lacked TILs only had a rate of $4.5 \%$. Additionally, the 5-year progression-free survival rates for patients whose tumors harbored or lacked TILs were 31.0 and $8.7 \%$, respectively. Follow-up studies revealed that the prognostic value is strongest for $\mathrm{CD}^{+}$cytotoxic $\mathrm{T}$ lymphocytes that are localized within the epithelial component of tumors, rather than the associated stroma, and that other factors associated with cyto- toxic T-lymphocyte activity are also associated with increased survival, e.g., IFN $\gamma$, IFNR, TNF $\alpha$ and MHC class I. By contrast, regulatory $\mathrm{T}$ cells, which suppress immune responses and maintain tolerance to self-antigens, are associated with decreased survival [2]. The functional phenotype and composition of TIL subsets further supports the notion that immune activation is associated with improved survival [3]. For instance, $\mathrm{CD}^{+}{ }^{+} \mathrm{CD} 103^{+} \mathrm{T}$ cells with an effector memory phenotype are present in large numbers in some high-grade serous ovarian cancers and are correlated with survival, without the need to discriminate their epithelial versus stromal location [4]. These provoking findings provide the basis for therapeutic applications that dramatically bolster the number and functional activity of endogenous immune cells in high-grade serous ovarian cancer.

Current immunotherapies for EOC fall into three broad categories: broad-acting immune checkpoint inhibitors and cytokines; therapeutic vaccines and the focus of this editorial, adoptive lymphocyte transfer. The infusion of T lymphocytes, referred to as adoptive cell therapy (ACT), has the potential to enhance antitumor immunity, augment vaccine efficacy and eradicate tumor, as observed in some tumor types. Adoptive transfer of TILs requires the isolation of $\mathrm{T}$ cells from fresh patient biopsy speci-
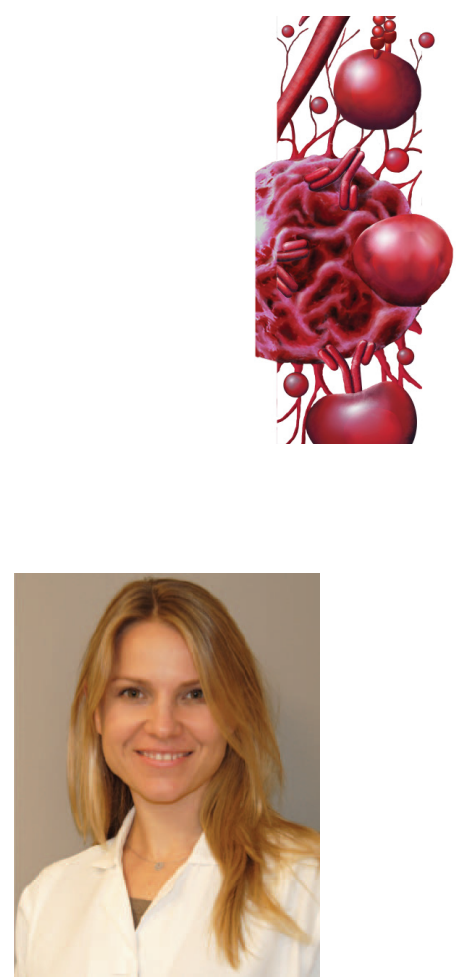

Katarzyna Urbanska Ovarian Cancer Research Center, Department of Obstetrics \& Gynecology, University of Pennsylvania, 3400 Civic Center Blvd, Bldg. 421, Philadelphia, PA 19104-5156, USA

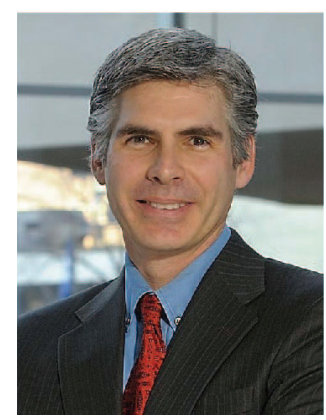

Daniel J Powell Jr

Author for correspondence: Ovarian Cancer Research Center, Department of Obstetrics \& Gynecology, University of Pennsylvania, 3400 Civic Center Blvd, Bldg. 421, Philadelphia, PA 19104-5156, USA and

Perelman School of Medicine,

Department of Pathology \& Laboratory Medicine, University of Pennsylvania, Philadelphia, PA, USA

Tel.: +1 2155734783

Fax: +1 2155735129

poda@mail.med.upenn.edu

Future $\because$ Medicine part of 
mens and the progressive expansion of tumor-specific $\mathrm{T}$ cells ex vivo. Early studies have identified methods to yield polyclonal tumor-reactive $T$ cells from tumors or ascites. In addition to TILs being present in ovarian cancer, the tumor cells themselves express individualized mutated self-proteins [5] and shared tumor associated-antigens, such as HER2/Neu, folate receptor alpha, mesothelin, NY-ESO-1, WT1 and TAG72 , which can serve as targets for humoral and cellular immune responses. Together, the characteristics of tumor cells and immune components in the EOC microenvironment suggest that these cancers may be responsive to adoptive immunotherapy.

Early clinical trials of ACT in EOC utilized autologous ex vivo activated peripheral blood $\mathrm{T}$ cells that were armed with a bispecific antibody, OC/TR, for redirected specificity for folate receptor alpha [6]. Patients received tumor debulking laparotomy followed by two cycles of five daily intraperitoneal infusions of armed $\mathrm{T}$ cells plus recombinant interleukin-2 (IL-2). Encouraging results were reported with an overall intraperitoneal response rate of $27 \%$, including three complete responses in the 19 patients evaluated. Importantly, no overt 'on-target, off-tumor' immune sequelae were observed in healthy organs that express low level folate receptor and thus established that autologous $\mathrm{T}$ cells with redirected specificity could induce tumor regression in EOC.

\section{"Evidence accumulated over the past two decades supports the notion that adoptive cell therapy may be a powerful treatment option for ovarian cancer patients. We stand at a transition point at which bench research has increasingly moved to bedside application."}

An alternative approach to T-cell transfer in EOC has relied on the use of endogenous tumor-reactive TILs derived from autologous resected tumor. In one study, patients who received adjuvant therapy with TILs following completion of chemotherapy and surgical debulking experienced a survival advantage with a 3-year disease-free survival rate of $100 \%$, compared with $67.5 \%$ in the cohort receiving chemotherapy alone, although these patients lacked available TIL and may have been in a poor prognostic group [1,7]. Since then, opportunities for optimization have become available to enhance the efficacy of ACT for EOC. For instance, host preconditioning with lymphodepleting chemotherapy increases the response to adoptive immunotherapy with TILs. Additionally, increased knowledge and optimized methods of tumor-reactive TIL selection and expansion can further bolster the ACT potency. For example, TIL persistence after infusion is associated with response to therapy and $\mathrm{T}$ cells that persist in patients experiencing tumor regression are characterized by a less-differentiated, $\mathrm{CD} 27^{+} \mathrm{CD} 28^{+}$ memory phenotype $[8]$, rationalizing the use of lessdifferentiated T cells in ACT. Moreover, we recently identified tumor necrosis factor receptor superfamily, member 9 (TNFRSF9, CD137, 4-1BB) as a marker for the identification of tumor-reactive $\mathrm{T}$ cells in tumors from EOC patients [9] and developed a rapid, comprehensive system to isolate tumor-reactive $\mathrm{T}$ cells with superior antitumor-potential in vitro and in vivo in a preclinical model of human EOC. Since this approach captures TILs with both shared and patient-specific antigen specificity indiscriminately, it is likely to bolster adoptive TIL efficacy in EOC, as emerging data suggests that $T$-cell reactivity against tumor-specific neo-antigens may be critical to the clinical efficacy of ACT therapy [10].

Some patients are not eligible to receive TIL ACT due to nonresectable disease or inability to produce tumor-reactive TILs. One approach to overcome this challenge is to either induce or engineer $\mathrm{T}$ cells to redirect them against tumor. Since several ovarian cancerassociated antigens have been identified, antigen-based vaccines have been utilized to induce tumor-reactive T cells. In Phase I trials, vaccination with overlapping long peptides from a single human cancer/testis antigen, NY-ESO-1, with poly-ICLC adjuvant was able to induce both $\mathrm{CD}^{+}$and $\mathrm{CD}^{+}{ }^{+} \mathrm{T}$-cell responses in most vaccinated patients [11,12]. Potent vaccine-primed $\mathrm{T}$ cells against an antigen such as NY-ESO-1 would appear amenable to ACT. Alternatively, one may apply a multiantigen vaccine comprised of known and private patient-specific antigens to broaden the antitumor response in ACT. In our study, HER2 peptide- and whole tumor lysate-pulsed autologous DC vaccination in combination with bevacizumab and metronomic cyclophosphamide, followed by adoptive transfer of autologous vaccine-primed $T$ cells, showed safety, and importantly, immunological and clinical efficacy in four out of six treated patients [13].

Engineering $T$ cells to redirect their specificity is another promising approach for treating EOC patients without available TILs. One route relies on genetically modifying nonreactive patient $\mathrm{T}$ cells ex vivo to express a cloned T-cell receptor (TCR) highly specific to tumor-associated epitope, thereby generating large numbers of therapeutic $T$ cells with tumor antigen-specificity conferred via introduction of the exogenous TCR. ACT therapy with TCRtransduced $T$ cells redirected against melanoma-differentiation can be effective in metastatic melanoma patients [14] and may be effectively applied to EOC using alternative TCRs. HLA-A2-restricted TCRs 
specific for epitopes from known ovarian cancer antigens such as NY-ESO-1, WT1, p53 are available for clinical application [15-17]. The field is now anticipating encouraging results from ongoing clinical trials with NY-ESO-1 TCR in EOC (NCT01567891). The NY-ESO-1 TCR was safe and effective in TCR-therapy for melanoma and synovial sarcoma [15]. Beyond NY-ESO-1, a new ErbB-2 ${ }_{369-377}$ peptide-specific TCR isolated from a ErbB2 peptide-pulsed DC vaccinated HLA-A2 ${ }^{+}$patient also holds promise for targeting ovarian cancer [18], given its broad expression of ErbB2 at immunologically detectable levels [19].

While TCRs can recognize intracellular processed antigen, MHC loss and loss of antigen processing machinery may thwart their ability to recognize some ovarian cancers. Alternatively, T cells can be transduced to express a chimeric antigen receptor (CAR) to permit recognition of surface antigens. CARs are composed of an extracellular domain that specifically binds to tumor epitopes through a single-chain variable fragment antibody linked to intracellular signaling domains that mediate T-cell activation. Because CARs bind to native cell surface tumor-antigens, they have two major advantages over TCRs. First, the HLA-independent recognition of antigen makes them broadly applicable regardless of the subject's HLA type and level of HLA expression on tumor cells. Additionally, incorporation of costimulatory domains, for example, CD27, CD28 and/or 4-1BB, into CARs supports activation, survival and persistence of engineered $\mathrm{T}$ cells [20]. Although, CARs specific for TAAs expressed by EOC have been investigated in mice and/or humans, for example, FR $\alpha$ [20], MUC-1, HER-2 [21] and mesothelin [22], clinical trials utilizing this approach are limited. To date, only one pilot study of adoptive transfer of CAR T cells has been conducted in ovarian cancer. Although, no clinically evident antitumor responses, feasibility and safety of CAR therapy in EOC was established [23], and the limitations of this pilot study have now been overcome. Persistence of engineered $\mathrm{T}$ cells can be dramatically improved by using human single-chain variable fragment and by adding costimulatory signaling domain to the CAR. With platform optimization, two new costimulated antimesothelin CAR T-cell trials are now being conducted in ovarian cancer; one involving the intravenous infusion of lentiviral transduced antimesothelin CAR

\section{References}

1 Zhang L, Conejo-Garcia JR, Katsaros D et al. Intratumoral T cells, recurrence, and survival in epithelial ovarian cancer. N. Engl. J. Med. 348(3), 203-213 (2003).

2 Curiel TJ, Coukos G, Zou L et al. Specific recruitment of regulatory $T$ cells in ovarian carcinoma fosters immune
T cells administered with and without preconditioning with lymphodepleting chemotherapy (NCT02159716) and another applying lymphodepleting preconditioning chemotherapy, and the transfer of the antimesothelin CAR T cells with Interlekin-2 cytokine administration (NCT01583686). Given the expression of mesothelin protein on peritoneal and pleural cavities and pericardial tissues, there is great interest in whether these CARs can differentiate between overexpressed antigen on cancer and that on healthy tissues to provide a safe and effective therapeutic window in women with advanced EOC.

Evidence accumulated over the past two decades supports the notion that ACT may be a powerful treatment option for ovarian cancer patients. We stand at a transition point at which bench research has increasingly moved to bedside application. Numerous studies have demonstrated the feasibility of adoptive transfer of natural or genetically engineered lymphocytes with TAA specificity as a method of treating cancer. As this concept undergoes further refinement, more effective and safe approaches will undoubtedly be developed, and novel combinations with additional immunomodulatory agents to augment the functionality of adoptively transferred $\mathrm{T}$ cells, such as cytokines, immune checkpoint inhibitors or agents that reduce immunosuppressive cell function and/or number, may ultimately increase ACT efficacy and aid in allowing ACT to reach its full therapeutic potential in women with ovarian cancer.

\section{Financial \& competing interests disclosure}

This work was supported by grants from the NIH (RO1CA168900) and the Joint Fox Chase Cancer Center and University of Pennsylvania Ovarian Cancer SPORE (P50 CA083638). DJ Powell Jr is a consultant to Lion Biotech, has received commercial research support from Novartis and has financial interests due to intellectual property and patents in the field of cell and gene therapy. Conflicts of interest are managed in accordance with University of Pennsylvania policy and oversight. The authors have no other relevant affiliations or financial involvement with any organization or entity with a financial interest in or financial conflict with the subject matter or materials discussed in the manuscript apart from those disclosed.

No writing assistance was utilized in the production of this manuscript.

privilege and predicts reduced survival. Nat. Med. 10(9), 942-949 (2004).

3 Milne K, Kobel M, Kalloger SE et al. Systematic anaᄀlysis of immune infiltrates in high-grade serous ovarian cancer reveals CD20, FoxP3 and TIA-1 as positive prognostic factors. PLoS ONE 4(7), e6412 (2009). 
4 Webb JR, Milne K, Watson P, Deleeuw RJ, Nelson BH. Tumor-infiltrating lymphocytes expressing the tissue resident memory marker CD103 are associated with increased survival in high-grade serous ovarian cancer. Clin. Cancer Res. 20(2), 434-444 (2014).

5 Wick DA, Webb JR, Nielsen JS et al. Surveillance of the tumor mutanome by $\mathrm{T}$ cells during progression from primary to recurrent ovarian cancer. Clin. Cancer Res. 20(5), 1125-1134 (2014).

6 Canevari S, Stoter G, Arienti F et al. Regression of advanced ovarian carcinoma by intraperitoneal treatment with autologous $T$ lymphocytes retargeted by a bispecific monoclonal antibody. J. Natl Cancer Inst. 87(19), 1463-1469 (1995).

7 Fujita K, Ikarashi H, Takakuwa K et al. Prolonged diseasefree period in patients with advanced epithelial ovarian cancer after adoptive transfer of tumor-infiltrating lymphocytes. Clin. Cancer Res. 1(5), 501-507 (1995).

8 Powell DJ, Jr, Dudley ME, Robbins PF, Rosenberg SA. Transition of late-stage effector $\mathrm{T}$ cells to $\mathrm{CD} 27^{+} \mathrm{CD} 28^{+}$ tumor-reactive effector memory $\mathrm{T}$ cells in humans after adoptive cell transfer therapy. Blood 105(1), 241-250 (2005).

9 Ye Q, Song DG, Poussin M et al. CD137 accurately identifies and enriches for naturally occurring tumor-reactive $\mathrm{T}$ cells in tumor. Clin. Cancer Res. 20 (1), 44-55 (2014).

10 Tran E, Turcotte S, Gros A et al. Cancer immunotherapy based on mutation-specific $\mathrm{CD} 4^{+} \mathrm{T}$ cells in a patient with epithelial cancer. Science 344(6184), 641-645 (2014).

11 Welters MJ, Kenter GG, Piersma SJ et al. Induction of tumorspecific $\mathrm{CD}^{+}$and $\mathrm{CD}^{+}{ }^{+} \mathrm{T}$-cell immunity in cervical cancer patients by a human papillomavirus type $16 \mathrm{E} 6$ and E7 long peptides vaccine. Clin. Cancer Res. 14(1), 178-187 (2008).

12 Sabbatini P, Tsuji T, Ferran L et al. Phase I trial of overlapping long peptides from a tumor self-antigen and poly-ICLC shows rapid induction of integrated immune response in ovarian cancer patients. Clin. Cancer Res. 18(23) 6497-6508 (2012).

13 Kandalaft LE, Powell DJ Jr, Chiang CL et al. Autologous lysate-pulsed dendritic cell vaccination followed by adoptive transfer of vaccine-primed ex vivo co-stimulated $\mathrm{T}$ cells in recurrent ovarian cancer. Oncoimmunology 2(1), e22664 (2013).
14 Morgan RA, Dudley ME, Wunderlich JR et al. Cancer regression in patients after transfer of genetically engineered lymphocytes. Science 314(5796), 126-129 (2006).

15 Robbins PF, Morgan RA, Feldman SA et al. Tumor regression in patients with metastatic synovial cell sarcoma and melanoma using genetically engineered lymphocytes reactive with NY-ESO-1. J. Clin. Oncol. 29(7), 917-924 (2011).

16 Cohen CJ, Zheng Z, Bray R et al. Recognition of fresh human tumor by human peripheral blood lymphocytes transduced with a bicistronic retroviral vector encoding a murine anti-p53 TCR. J. Immunol. 175(9), 5799-5808 (2005).

17 Stauss HJ, Thomas S, Cesco-Gaspere M et al. WT1-specific $\mathrm{T}$ cell receptor gene therapy: improving TCR function in transduced T cells. Blood Cells Mol. Dis. 40 (1), 113-116 (2008).

18 Lanitis E, Smith JB, Dangaj D et al. A human ErbB2specific T-cell receptor confers potent antitumor effector functions in genetically engineered primary cytotoxic lymphocytes. Hum. Gene Ther. 25(8), 730-739 (2014).

19 Lanitis E, Dangaj D, Hagemann IS et al. Primary human ovarian epithelial cancer cells broadly express HER2 at immunologically-detectable levels. PLoS ONE 7(11), e49829 (2012).

20 Song DG, Ye Q, Poussin M, Harms GM, Figini M, Powell DJ Jr. CD27 costimulation augments the survival and antitumor activity of redirected human $\mathrm{T}$ cells in vivo. Blood 119(3), 696-706 (2012).

21 Zhao Y, Wang QJ, Yang S et al. A herceptin-based chimeric antigen receptor with modified signaling domains leads to enhanced survival of transduced $T$ lymphocytes and antitumor activity. J. Immunol. 183(9), 5563-5574 (2009).

22 Beatty GL, Haas AR, Maus MV et al. Mesothelin-specific chimeric antigen receptor mRNA-engineered $\mathrm{T}$ cells induce anti-tumor activity in solid malignancies. Cancer Immunol. Res. 2(2), 112-120 (2014).

23 Kershaw MH, Westwood JA, Parker LL et al. A Phase I study on adoptive immunotherapy using gene-modified $\mathrm{T}$ cells for ovarian cancer. Clin. Cancer Res. $12(20 \mathrm{Pt} 1)$, 6106-6115 (2006). 